\title{
A Pragmatic Introduction to Chaos Theory for Engineers
}

\begin{abstract}
Highly repetitive mechanical systems like those present in industrial machinery, are prone to undergo chaos. To understand this, engineers responsible for the operation of machinery systems, must be aware of the "Routes to Chaos". This work presents the results of an investigation carried-out in a Virtual Laboratory, created to investigate the transition to chaos in oscillating (rotating) systems, immersed in a dampening medium (impossible to avoid damping in a non ideal situation), under the action of an applied force (a motor).
\end{abstract}

KeYwords: Virtual Lab, simulation, non-linear systems, oscillations, chaos.

A Pragmatic Introduction to Chaos Theory FOR ENGINEERS

\section{RESUMEN}

Los sistemas mecánicos altamente repetitivos, como aquellos presentes en las máquinas industriales, son factibles de experimentar caos. Para entender esto, los ingenieros responsables del funcionamiento de un sistema de maquinarias deben estar al tanto de las "Rutas hacia el Caos". Este trabajo presenta los resultados de una investigación llevada a cabo en un Laboratorio Virtual, creado para estudiar la transición al caos en sistemas oscilantes (rotativos) en un medio amortiguador (imposible evitar la amortiguación en un medio no ideal), bajo la acción de una fuerza aplicada (un motor).

Palabras clave: Laboratorio virtual, simulación, sistema no-lineal, oscilaciones, caos.

\section{INTRODUCTION}

Consider the case of a sewing machine, the needle goes up and down at a particular frequency, the traction of the cloth takes place at another frequency, and the pulling of the thread is made at even other frequency. This is just an example of a mechanical system where the three mentioned frequencies must operate in harmony for a good performance of the machine. According to the theory of chaos, systems with competing frequencies are highly prone to undergo chaos. Mechanical systems have not been built to experiment chaos and hence, they collapse before reaching that phase.

Repetitive mechanical systems like those present in industrial machinery, consist of a series of oscillating (rotating) sub-systems, each one having its own frequency of oscillation (rotation), and mostly all of them (if not all) interacting, hence from the point of view of chaos theory, these systems are strong candidates to experience a competence between frequencies, the main ingredient to chaos.

Facing the above described scenario it is very important for an engineer to have notions of oscillations and to be aware of their extreme behavior, namely, chaos.

The formal study of oscillations is based on models, being the simplest ones the swinging pendulum and the oscillating spring, both in vacuum (free oscillators). However, free oscillating pendulums and springs are rather ideal linear oscillators. Real life oscillatory mechanisms are to some extent non-linear and subject to some kind of friction (damping). Besides this, usually there is also an external force acting upon these systems, so as to maintain its motion. Forced oscillating systems include at least two oscillating frequencies, one corresponding to the oscillator itself (its natural frequency) and another, associated to the external applied force. Hence there are -in the simplest case- two competing frequencies. It is well-known that after some initial time the oscillator winds up oscillating with the frequency of the external applied force. It is also known that dissipative systems with two competing frequencies exhibit transitions to chaos $^{3,10}$

Resonance is common in oscillatory systems and when designing oscillatory mechanisms, engineers try by all means to avoid

VirtualDynamics- Virtual Labs: Science \& Engineering. Calle Catorce, $\mathrm{N}^{\circ} 572$, Las Magnolias de Surco, Lima 33, Perú. Director@VirtualDynamics.org --- www.VirtualDynamics.org 
that so as to keep away from undesired harmful outof-control vibrations which may eventually wreak havoc.

Under some conditions non-linear oscillators may display irregular and really complex oscillations, being it impossible to detect its period and amplitude of oscillation, in other words, the oscillation becomes aperiodical (it never exactly repeats).

Since the 1980's researchers have made enormous efforts to understand aperiodic motions of oscillatory systems, these endeavors have resulted in the Chaos Theory ${ }^{1,10}$. Aperiodicity of non-linear dynamical systems is also known as Chaotic Dynamics. Chaos theory would had been fathered by Henri Poincare in the 1890's, but he had no computers.

Back in the 1980's when chaos research started, computers were not as powerful and friendly as they are today, this author used to work then under time-sharing mode in mainframes like IBM 370 and IBM 4341, and generating data to make plots like those shown in Figs 2 through 4 was not as easy and quick as it is today.

Concerning the organization of this paper, next an introduction to chaos is presented, then a description of the created software is exposed and after that the application to the case of the damped nonlinear oscillator with variable damping is reported.

\section{COMMON CHAOS VS PHYSICAL CHAOS}

While everyday language understands chaos as complete disorder, tantamount to total randomness, it has a much subtler meaning in physics.

Chaos is the phenomena related to the occurrence of randomness and unpredictability in completely deterministic systems, it is not to be equated simply with disorder, it is more appropriate to consider Chaos as a kind of order without periodicity ${ }^{2}$.

Stochasticity, Dynamical Chaos or simply Chaos ${ }^{7-8}$, is the appearance of apparently random motion in a deterministic dynamical system, this is, a system with no random forcing.

Even at long times chaotic systems include elements of order, in other words, chaos is not completely random ${ }^{8-9}$.

A chaotic system is not a random system, it is deterministic. A chaotic system seems random if the beholder fails to recognize that it is chaotic. In a random system it is impossible to determine future states from previous states, in deterministic systems, that prediction is possible, provided the system is not sensitive to small variations in initial conditions.

\section{PERIOD DOUBLING ROUTE TO CHAOS}

Several routes to chaos have been observed, however, the Period Doubling Route to Chaos $^{4-5}$ is a universal and fundamental form of transition from periodicity to chaos (Transition to chaos ${ }^{6}$ ), observed in many mathematical and real systems.

In the Transition to Chaos $^{6}$, a system evolves toward non periodic time dependence as one or more parameters are varied.

In the Period Doubling ${ }^{6}$ process, the time it takes the system to repeat itself, this is its Period, doubles and then doubles again and again, until the period becomes essentially infinitely long, as one or more control parameters (temperature, velocity, force, etc) are varied. Hence the Period Doubling Route to Chaos involves an infinite sequence of Bifurcations $^{6}$, which appears as a Cascade of Bifurcations. All systems that become chaotic by the period doubling process do so in the same universal way. Beyond the period doubling cascade the stable periodic orbit disappears and chaos dominates the scene. However it is important to mention that not all systems evolving to chaos follow the bifurcation cascade behavior.

In mechanical systems, the above mentioned control parameter is usually, the temperature, whose values are not easy to maintain within a convenient range, which -to worsen the situation- becomes smaller as the machine degrades by its continuous use.

\section{STRANGE ATTRACTOR}

A strange attractor ${ }^{6}$ is the shape (geometrical object) depicted in Phase Space (a.k.a. State Space) by a chaotic system. These attractors are fractal (have non-integer dimension) and consist of an infinitely number of closely spaced layers. Nearby trajectories diverge from each other by an amount that is exponential in time rather than proportional to time.

\section{DEPENDENCE ON INITIAL CONDITIONS}

A quality that characterizes chaotic systems is that these definitively show a sensitive dependence on 
initial conditions. Other systems may or may not exhibit this sensitivity, but chaotic ones definitively do. Sensitivity to initial conditions however, does not automatically entail chaos.

\section{STUDYING CHAOS WITHOUT COMPUTERS}

It would be impossible to construct a mechanical system capable of going through a cascade of period doubling bifurcations; any mechanical system would collapse shortly after the first period doubling. For this reason the study of the transition to chaos must be made with computers. Some researchers have attempted the study of chaos by simulating oscillators, with electrical circuits.

\section{THE VIRTUAL LAB}

A Virtual Lab to numerically solve by the RungeKutta algorithm a second order non linear differential equation has been developed, this software can evaluate the equation in up to one million $\left(10^{6}\right)$ time steps, it shows the Time Series and the Phase Diagram (Phase Space) and detects and plots on screen the peaks and valleys of the amplitudes, all in a single screen and taking no more than 6 seconds in a Dual-Core Processor PC. Additionally a pendulum in motion according to the data generated in the simulation may be appreciated at the click of a button. This pendulum however, is just to visualize the oscillations; the problem under study may have nothing to do with a pendulum.

The two most important products of this software are the plotting on screen of the Bifurcation Cascade route to chaos (Fig. 4) of the equation it is solving and the animated pendulum, in this way, the route to chaos and chaos itself are easily and quickly visualized.

Seeing that dealing with non-linear systems is not straightforward at all, determining the parameters that generate the transition to chaos for a given differential equation is not an easy undertaking, and one way of detecting them may be by trialand-error. If this program were set to operate on a trial-and-error mode search for chaos over randomly generated parameters, it would be able to investigate up to 20 to 30 different cases every minute (a huge time saving), and by virtue of a pattern recognition technique operating on phase space, automatically might report chaos when encountered.

\section{CONSTRUCTING THE MATHEMATICAL MODEL OF THE OSCILLATOR}

In order to investigate the transition to chaos in the Non-Linear Damped and Forced Oscillator, its mathematical model must be constructed.

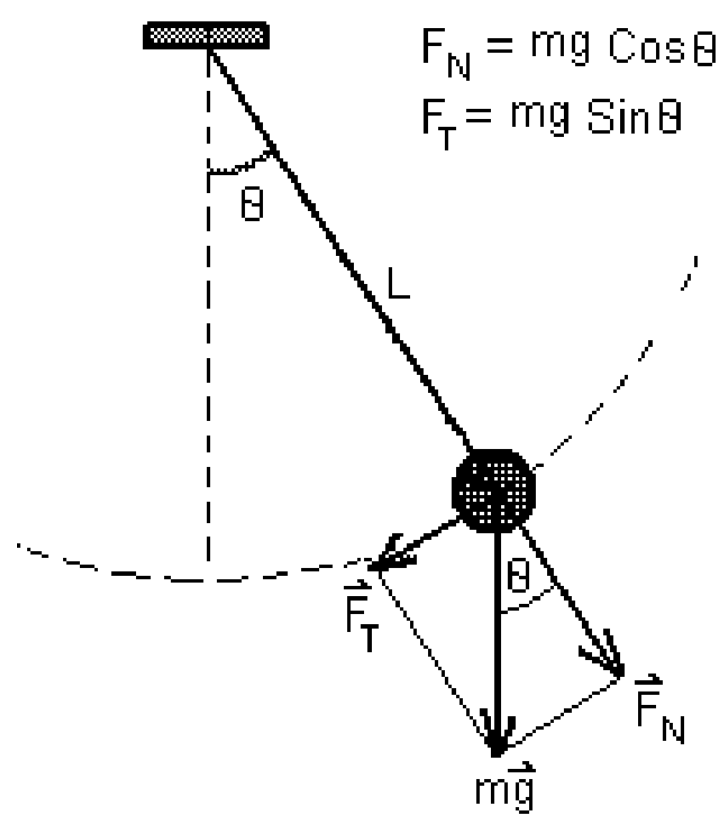

Fig. 1. An object oscillating in a variable damping medium.

Consider an oscillator in the form of a pendulum of mass $m$ and length $L$. According to Newton's second law, the equation of motion of the pendulum is

$m L \frac{d^{2} \theta}{d t^{2}}=-m g \sin \theta$

where the second time derivative of the displacement is the tangent acceleration of the pendulum. The frequency of oscillation of the free pendulum

is $\omega_{o}=\sqrt{g / L}$ which is known as its "Natural Frequency of oscillation". In the case of very small oscillations (less than $15^{\circ}$ ), this oscillator executes a Simple Harmonic Motion.

If the pendulum were oscillating in a viscous medium offering a resistance proportional to the velocity, the equation would become

$m L \frac{d^{2} \theta}{d t^{2}}=-m g \sin \theta-b \frac{d \theta}{d t}$

where the first time derivative of the displacement, is the velocity, the minus sign means that the damp- 


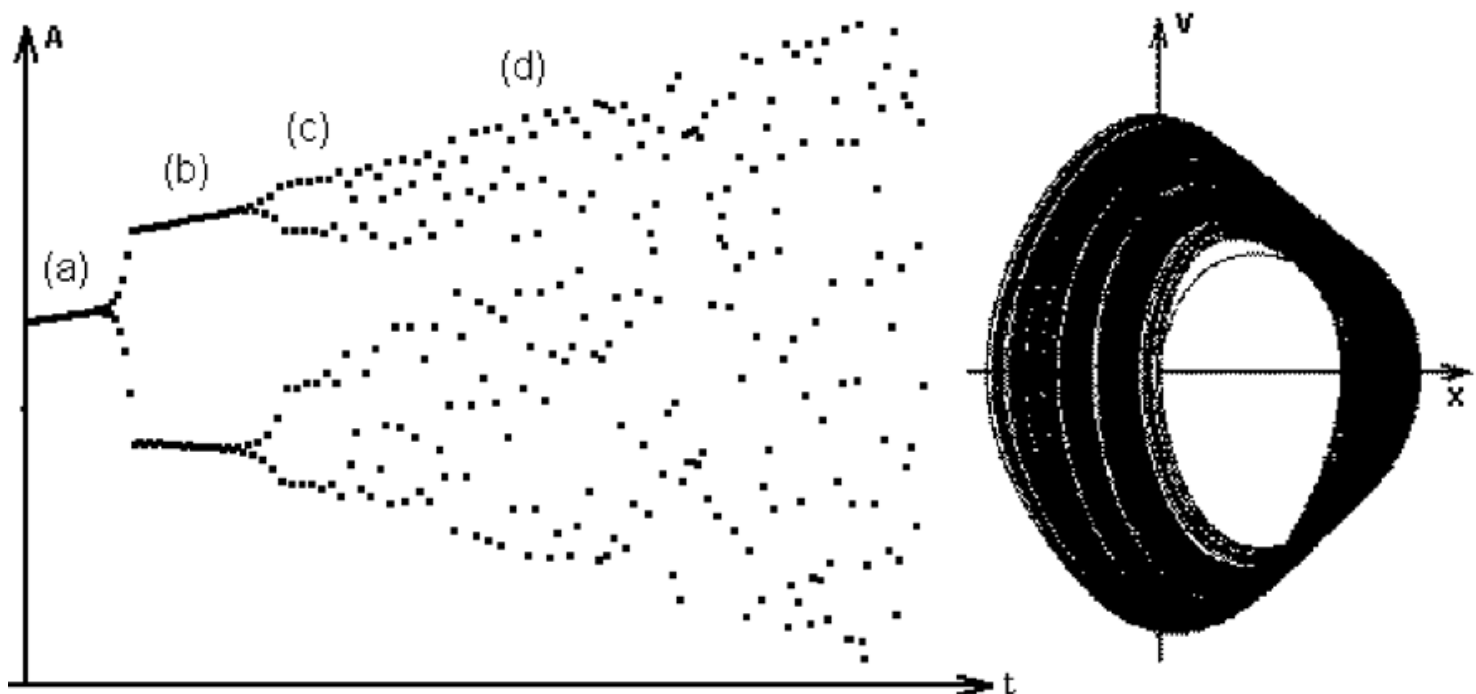

Fig. 2. Amplitude peaks vs time: Two bifurcations are clearly seen, before chaotic motion sets in. The corresponding Phase Diagram (velocity vs position) is also shown.

ing force is against the direction of motion, and $b$ is a constant of proportionality, related to the viscosity of the medium.

If additionally, an external oscillating force $F_{0}$, with frequency $\Omega$, drives the pendulum, the equation of motion turns out to be

$m L \frac{d^{2} \theta}{d t^{2}}=-m g \operatorname{Sin} \theta-b \frac{d \theta}{d t}+F_{0} \operatorname{Sin} \Omega t$

This equation is usually written as,

$\frac{d^{2} \theta}{d t^{2}}+\left(\frac{b}{m L}\right) \frac{d \theta}{d t}+\omega_{o}^{2} \sin \theta=\left(\frac{F_{\theta}}{m L}\right) \sin \Omega t$

which is the equation of motion of a non-linear damped and forced oscillator immersed in a viscous medium.

Equation (1) which is a second-order differential equation, models a non-linear oscillator ${ }^{2,3}$ (a pendulum in the simplest case) immersed in a dissipative medium of damping factor $b$ and which is connected to an external applied sinusoidal force $F_{\text {o }}$ oscillating

with frequency $\Omega$, being $\omega_{o}$ the angular frequency of the free linear oscillator (without damping).

In a variable damping medium, one that either becomes thicker or thinner with time, the factor $b$ in equation (1), changes as

time passes by.

It is expected that after a transient stage, the oscillator oscillates with the frequency $\Omega$ of the applied external force.

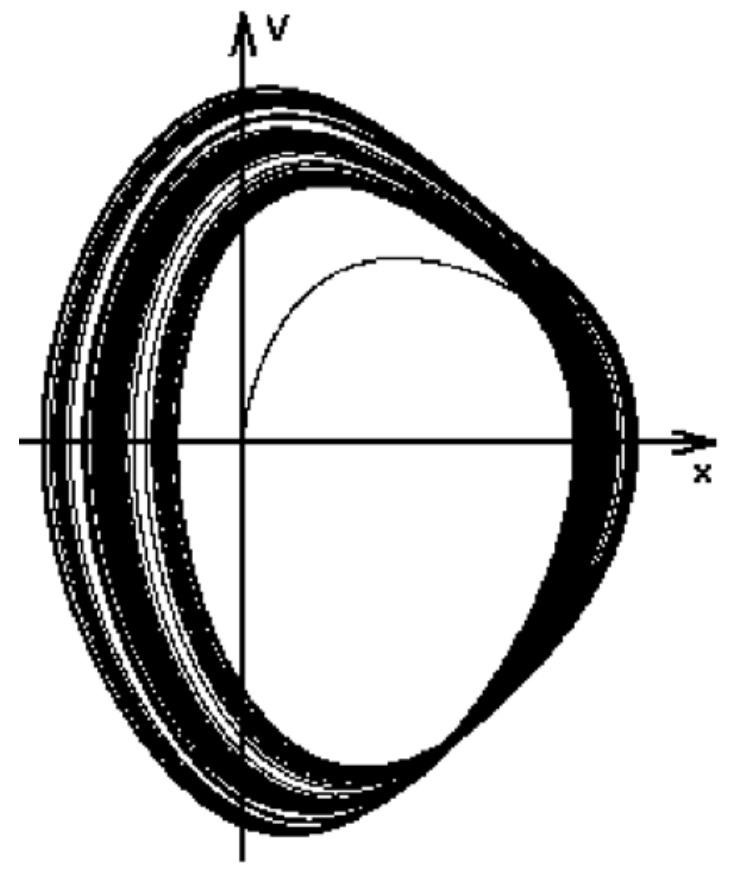

Fig. 3. Phase Diagram for the first 530000 (x, $V)$ points of the simulation corresponding to Fig. 2, before the onset of chaos.

In view of the fact that the system under investigation undergoes dissipation (damping $b$ ), and since there are two competing frequencies, it is expected to observe a transition to chaos ${ }^{3}$. In the investigation reported in this document, the applied force Fo was slowly increased, which is equivalent to slowly lowering the damping. 


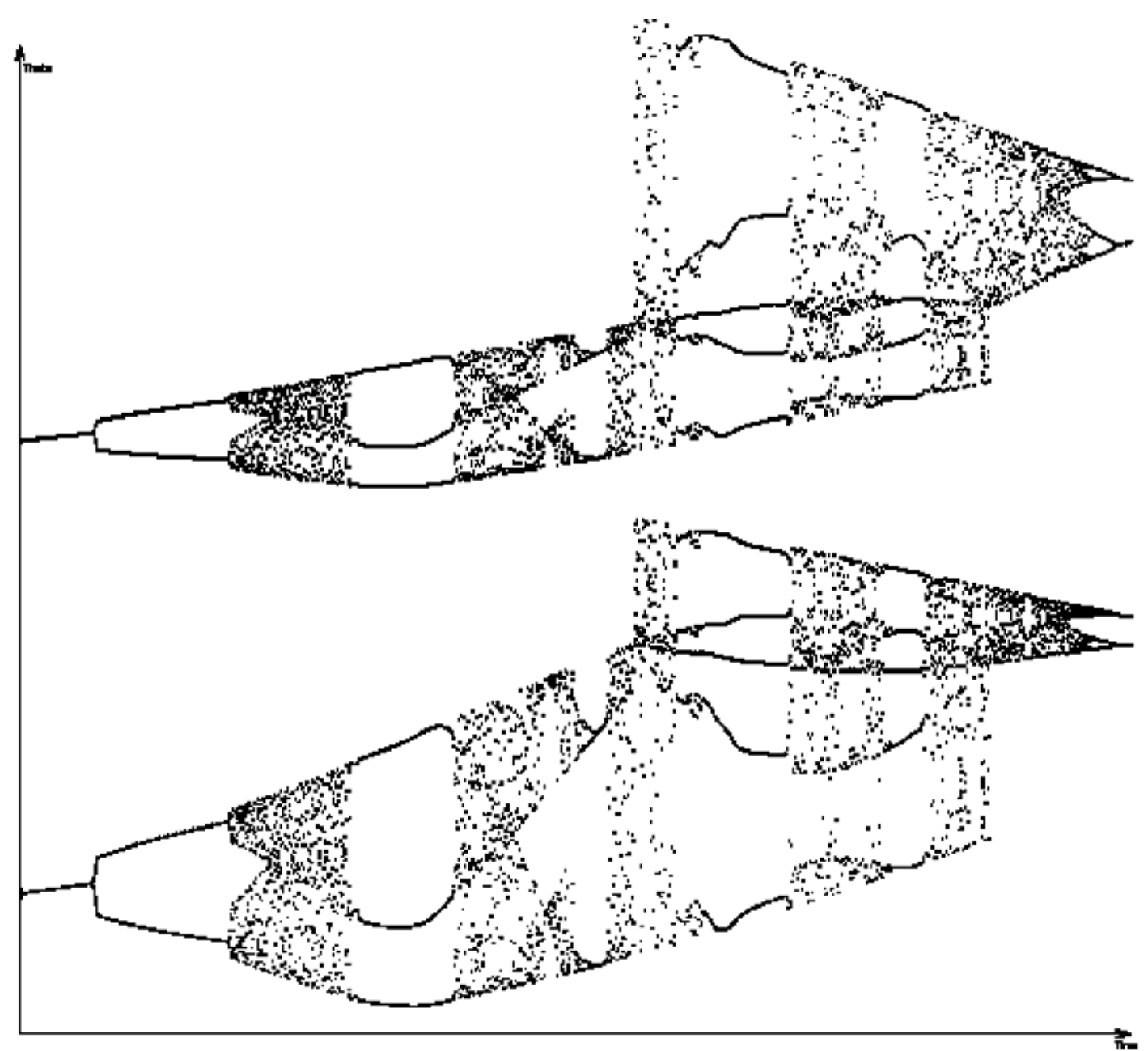

Fig. 4. Bifurcation cascade of the non-linear damped and forced oscillator. The graph shows the oscillation amplitude peaks (top) and valleys (bottom) versus time, for 1000000 time steps while applied force increases.

\section{RESULTS OF THE INVESTIGATION}

Based on both experience and physical intuition, the set of parameters where the oscillator under study shows a transition to chaos was successfully determined with the help of the developed software and after just a few trials. The resulting cascade of bifurcations is shown in fig. 2, corresponding to 900,000 time steps. This figure also displays the corresponding phase diagram (plot of velocity $\mathrm{V}$ versus displacement $X$ ). In Fig 2, region (a) corresponds to a period 1 , the regular situation. Region (b) corresponds to the first bifurcation, with period 2. In region (c) a second bifurcation takes place, with period 4 , and this is followed by region (d) with widespread chaos. There must be another bifurcation right after region (c) but it is not evident with the plotting resolution used.

Fig. 3 shows the phase diagram for the first 530000 time steps of the simulation shown in Fig. 2, this is, from the start up to the point where the two first bifurcations in Fig. 2 are still easily distinguishable, right before the onset of chaos. It can be seen that the graph has self similarity, hence it may be a fractal.

Fig. 4 displays the oscillation amplitude peaks and valleys for a simulation with equation (1) along one million time steps, as the applied force increases. The cascade of bifurcations is clearly seen.

Mechanical systems are constructed to operate under a given frequency, which corresponds to region (a) in Fig. 2. This frequency may gradually change a little due for example, to an increase in temperature. When the system starts to alternate between two different frequencies (region $b$ in Fig.2), it is escaping its normal (expected) behavior and this must be understood as an alarm signal. A mechanical system, usually collapses before reaching region (c) in Fig. 2, this may be 
the reason why chaos is not in fact observed at these systems.

Fig 5 shows the Return Map for the case shown in Fig. 4. The Return Map is a plotting of $X_{n+1}$ as a function of $X_{n}$, where $X$ is the oscillation amplitude peak. It can be seen that for some values of $X_{n}$ , there are several possible values of $X_{n+1}$, which means that it is impossible to predict an amplitude of oscillation.

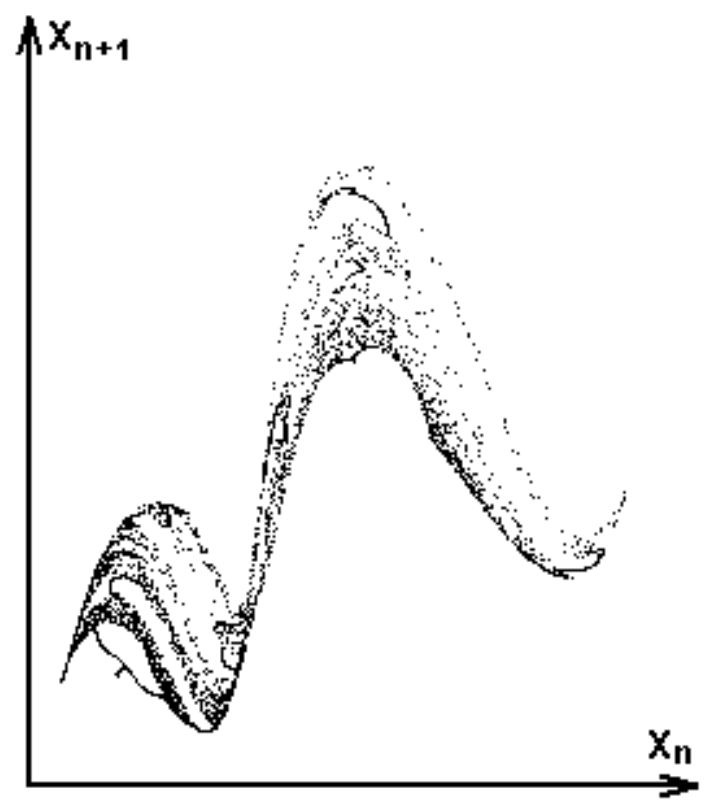

Fig. 5. Return Map for the case shown in Fig. 4. Notice that it is impossible to predict an amplitude of oscillation $X n+1$, from the previous amplitude $X n$, because there are several possible values of $X n+1$ for most $X n$.

\section{CONCLUSSIONS}

An introduction to Chaos Theory having the nonlinear forced and damped oscillator as a model, has been presented. The mathematical model, whose construction has been shown, was used as a simulation algorithm in a Virtual Lab spe- cially designed to solve -by means of the RungeKutta method- second order non-linear differential equations. The created software allows for a very quick simulation and visualization, reporting the Bifurcation Diagram, the Return Map, The Phase Diagram, and a visualization of the oscillations of the system under study.

\section{REFERENCES}

[1] Gonzales-Miranda, J.M. (2004). Synchronization and Control of Chaos, an Introduction for Scientists and Engineers. Imperial College Press, London.

[2] Chaos, Hao Bai-Lin, (1985). Preprint.

[3] Bak, P., Bohr, T., Mogens, H. Jensen (1985). Mode-locking and the transition to chaos in dissipative systems. Physica Scripta, Vol T9, pp 50-58

[4] Feigenbaum, M. (1978). Quantitative universality for a class of nonlinear transformations. J. Stat. Phys.

[5] Feigenbaum, M. (1983). Universal behavior in nonlinear systems, Physica D 7.

[6] Chaos, A glossary. Schewe, Phillip. American Institute of Physics. 1985

[7] Chernikov-Sagdeev-Zaslavsky (1968). Chaos: How regular can it be? Physics Today, Nov. 1988.

[8] Taubes, G. (1984). The Mathematics of Chaos, Discover, Sept. 1984.

[9] Zaslavsky, G.M. (1999). Chaotic Dynamics and the Origin of Statistical Laws, Physics Today, August 1999.

[10]Hilborn R.C., Chaos and Nonlinear Dynamics, Oxford Univ. Press. Preprint. 